\title{
Categorical Description of Simple-Folding a Map
}

\author{
Yiyang JIA ${ }^{\mathrm{a}, 1}$, Yan ZHAO $\mathrm{Z}^{\mathrm{b}, 2}$, Chao ZHANG ${ }^{\mathrm{c}}$

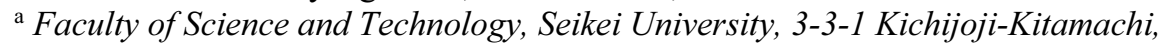 \\ Musashino-shi, Tokyo 1808633, Japan \\ ${ }^{\mathrm{b}}$ School of Computer Science and Communication Engineering, Jiangsu University, \\ Jiangsu Province 212013, China \\ ${ }^{\mathrm{c}}$ Faculty of Engineering, University of Fukui, Fukui-shi, Japan
}

\begin{abstract}
To compensate for the lack of abstraction in the field of origami, in this paper, we propose a categorical description that can be introduced to map folding. Specifically, we use a particular expression to abstract the folding process of a map with logical matrices. When the folding operations are restricted to two certain kinds, the simple folds and the simple unfolds, we can define categories of partly folded states of the map as poset categories. The property of posets induces many general categorical concepts, such as (co)product, opposite category, direct system, and so on. We then introduce how these general concepts are specified in the proposed categories. These conceptions and specifications brought us the hope to solve and study the map folding with contemporary mathematical methods, such as the (co)homology. Furthermore, our categorical description can potentially be applied to a more generalized version of the map folding, the flat-folding.
\end{abstract}

Keywords. Category theory, map folding.

\section{Introduction}

The category theory has been developed as a strong tool in many conventional studies and research fields during the previous several decades, and it has contributed to address many specified problems. The categorical description of singular (co)homology [1], the rephrase of sheaves and schemes in modern algebraic geometry [2], and the unification of usual manifolds and states studied in quantum field theory and quantum computing [3] are all notable examples. In the subject of geometric folding, however, such a powerful tool has never been presented. The intricacy may be equated to folding a paper with faces of various forms and sizes, each of which is extremely dependent on the metric, which is often not chasable by a categorical analysis.

Fortunately, in map folding, where the folding of a regular grid pattern of size $m \times n$ with a Mountain-Valley assignment (an MV assignment, in which every edge is assigned

${ }^{1}$ Corresponding Author, Yiyang JIA, Faculty of Science and Technology, Seikei University, 3-3-1 Kichijoji-Kitamachi, Musashino-shi, Tokyo 1808633, Japan; Email:yiyangjia1995@foxmail.com

2 Corresponding Author, Yan ZHAO, School of Computer Science and Communication Engineering, Jiangsu University, 301 Xuefu Road, Zhenjiang, Jiangsu Province 212013, China; Email:yanzhao_cs@ujs.edu.cn 
as either a "Mountain" or a "Valley" to specify the direction to fold) is desired, the concept of metric is weakened while the topology is strengthened. This allows us to use category theory instead of standard algorithmic methods to detect the properties of map folding in a highly abstracted fashion. In the next section, we will go through some existing results of map folding.

To simplify the map folding problem, Arkin et al. presented the simple folding model [4]. They explored three types of simple-folding models: one-layer simple folding, alllayers simple folding, and some-layers simple folding. Among these, the last one is the most generalized one, and it is utilized in this research as well.

We also introduce simple unfolding in order to construct the opposite category [5]. Every step of a (some-layer) simple folding (simple fold) folds some top or bottom layers along a single line to achieve a state that is also flat, as shown in figure 1, whereas every step in a simple unfolding (simple unfold) is an inverse operation of a simple fold.

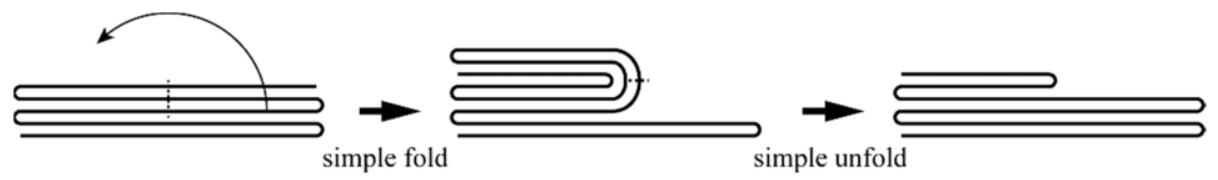

Figure 1. An example of simple fold and unfold.

On the other hand, logical matrices, which are employed as a representation in our categorical analysis of map folding, are also essential in current quantum computing research [3]. These matrices are seen as a natural representation of the category of relations, Rel. Some sophisticated categories, such as the category of Hilbert spaces Hilb and the category of $(n-1)$-dimensional compact oriented manifolds $\mathbf{n C o b}$, are introduced as nothing more than refined versions of Rel in [3].

The 0 and 1 in the logical matrices, on the other hand, can be thought of as degenerated cases of general probabilities with a range of 0 to 1 . From this perspective, the overlap of layers in a flat-folding, i.e., any flatly folded state of a sheet of paper with an arbitrary pattern, might theoretically be represented by numbers ranging from 0 to 1 . 0 indicates that they "do not overlap at all", while 1 indicates that they "completely overlap". The overlap relation of any two arbitrary layers must correspond to these two in our definition of simple-folding a map, thus either 0 or 1 . It is possible to generalize our map folding description to other flat-foldings.

We use a logical matrix representation to offer a categorical description of simplefolding a map in this work. We define the category of partially flatly folded states (middle states) of the map during simple folding, PS and built a one-to-one relationship between PS and the logical matrix representation category LM. In addition, we define notations such as opposite category, (co)product, and direct system in PS and LM.

\section{Background and Preliminaries}

In this section, we will introduce some related works of map folding, flat-folding, and simple-folding.

Map folding is a specific type of flat-folding. As mentioned in the last section, faces do not have to be exactly the same shape in most flat-foldings. It was shown that deciding whether such generalized patterns have flatly folded states is NP-hard [6]. This problem 
is still unsolved for map folding, although some results for low-dimensional map folding are given in $[4,7]$.

Despite this intractability of general flat-folding, the problem degenerates into a linear-time solvable scenario when constrained to a small enough area around a single vertex [8]. In other words, if the pattern has only one vertex, whether it can be folded to a plane is linear-time decidable. The purpose of emphasizing the different results involving local and global flat-foldability is because they might be related to "local-vsglobal" topics in cohomology theories. So far, studies on flat-foldings have only focused on local and global situations separately rather than the connection between them. However, the connection seems to be a much more intrinsic and complex subject from the categorical viewpoint.

As afore-mentioned, the simple folding and the simple unfolding were firstly introduced in [4] and [5], respectively. In [4], they used two kinds of special simple folds, crimps and end-folds, as the basic operations in a map of size $1 \times n$. An end-fold in a 1D map is a fold at either the first or last crease point, as shown in figure 2 . The distance between the last crease point and the corresponding end of the map is not larger than the distance between the adjacent crease points. A crimp is a fold made between two adjacent crease points marked with the letters $M V$ or $V M$. The length of the interval between the two crease points is locally minimum.

These two operations can be easily generalized to 2D maps of size $m \times n$ if we generalize the folded points in figure 2 to a line segment going through the rectangular paper along grid lines. According to one of our recent studies, any middle state (refers to a partly flatly folded state) of a map accessible by a simple folding can be accessed by a sequence of crimps and end-folds while respecting the rule that once two squares of the map touch each other, they would not be apart again [9].

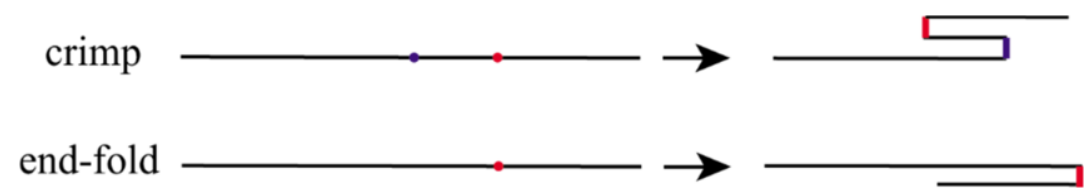

Figure 2. Two operations in the simple folding: crimp and end-fold. The red points indicate mountains and the blue point indicates a valley.

\section{Logical Matrix Representation}

Matrix of a map The $m \times n$ squares in a map can be indexed from 1 to $m n$. We then build a $m n \times m n$ matrix $M$ to represent the map. An element at the $i$-th column and the $j$-th row is denoted $m_{i j}$. It represents the relation between square $i$ and square $j$.

Adjacent relation In any middle state of the map, a pair of squares either touch each other or not. When two squares touch each other, we say that they are in an adjacent relation. For a pair of adjacent squares $i$ and $j$, there are two possible "up-down relation", i.e., $i$ is either below $(i<j)$ or above $j(j<i)$. For the consistency, we assign 1 to $m_{i j}$ if $i$ is supposed to be below $j$ in the final state, and assign 1 to $m_{j i}$ if $i$ is supposed to be over $j$ in the final state. Even though the "up-down relation" in a middle state could be different from the one in the final state, it can be easily computed following both the parity of the Manhattan distance between square $i$ and the first square and which side 
(the front side or the backside) $i$ faces up in the middle state. Other elements of the matrix would retain 0 . As illustrated in figure 3 . For this map of size $2 \times 4$, its corresponding matrix is an $8 \times 8$ matrix. The initial matrix is a zero-matrix, corresponding to (1). Then, the middle state (2) corresponds to the matrix below it, where $m_{43}$ and $m_{78}$ are assigned 1. When folded to the middle state in (3), $m_{51}, m_{26}$, and $m_{84}$ are assigned 1 . In the same manner, states in (4) and (5) are matched with the matrices below them, respectively.

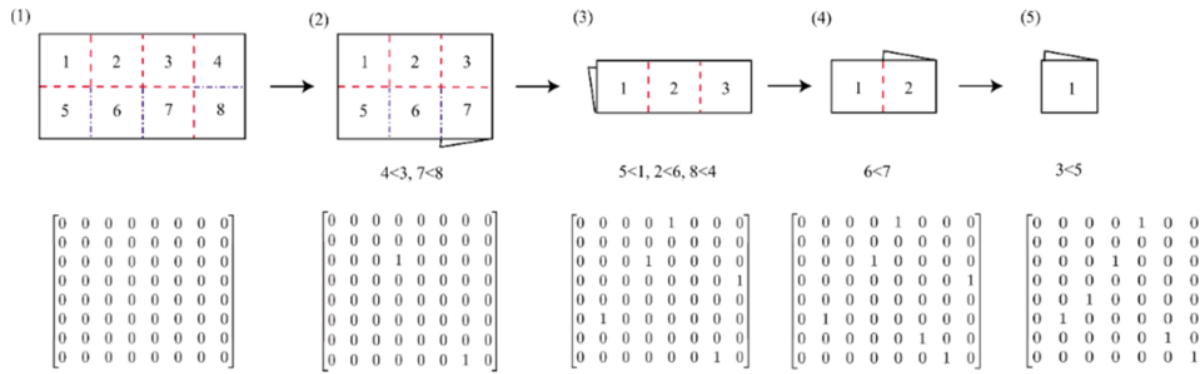

Figure 3. A simple folding of a map of size $2 \times 4$ and the adjacent relations assigned at every step of the folding.

We must mention that, although we only recorded the adjacent relations in the matrix, the entire "up-down relation", also involving the non-adjacent ones, can be computed as the transitive closure of the matrix. As an example, we give the computation of the transitive closure $C_{5}$ for the matrix of the final state $M_{5}$ (figure 3(5)) in formula (1). More introductions of the transitive closure can be found in [10].

$$
C_{5}=\sum_{i=1}^{\infty} A_{5}^{i}=\left[\begin{array}{llllllll}
0 & 1 & 1 & 1 & 1 & 1 & 1 & 1 \\
0 & 0 & 0 & 0 & 0 & 0 & 0 & 0 \\
0 & 1 & 0 & 1 & 0 & 1 & 1 & 1 \\
0 & 1 & 0 & 0 & 0 & 1 & 1 & 1 \\
0 & 1 & 1 & 1 & 0 & 1 & 1 & 1 \\
0 & 1 & 0 & 0 & 0 & 0 & 0 & 0 \\
0 & 1 & 0 & 0 & 0 & 1 & 0 & 0 \\
0 & 1 & 0 & 0 & 0 & 1 & 1 & 0
\end{array}\right]
$$

In a transitive closure $C$ of any middle state $M$, the element $c_{i j}$ means that square $i$ is supposed to be below square $j$ in the final state, but not necessarily adjacent. Because of the uniqueness of adjacency relations in a middle state, a logical matrix representation for each middle state is unique. A logical matrix, on the other hand, can only represent one intermediate state at a time.

\section{Categorical Analysis of the Map Folding}

In this section, first, we will define the two categories we will be looking at in this study. The first is the category of simple-folded middle states of a map of size $m \times n$, while the second is the category of their logical matrix representations. Following that, we will define some common category concepts in these categories. 


\subsection{Two categories}

The first category is PS, the category of middle states of a map by simple folds. All the conceivable middle states and final states of a map $M$ are objects in this category, and all the possible foldings (unfoldings excluded) between these states are morphisms. The identity morphism is defined as an empty folding that does not have any practical folding operation.

The second category, the category of the logical matrix representations, is denoted LM. The natural addition operation, inherited from the semiring $\Omega=(\{0,1\},+, \cdot)$, is utilized to construct morphisms, and all the logical matrices that are allocated in terms of the adjacent relations in each middle state are taken as objects. The identity morphism in this category is addition by the 0 matrix, which is a reassignment of the matrix elements with no significant changes. The number 0 denotes the identity morphism. Now, we can build a one-to-one correspondence, or we say, a one-to-one functor between PS and LM.

According to our fundamental setting of the folding operations, every time a crimp or an end-fold is folded, it connects two middle states (including the final states). Let's write $A, B \in O b(\mathbf{P S})$ for two arbitrary middle states, and $f \in \operatorname{Mor}(\mathbf{P S})$ for the crimp or the end-fold. As a result of $f$, certain squares will become adjacent and, as previously mentioned, will never be separated again. These adjacent relations correspond to a few more $1 \mathrm{~s}$ in the logical matrix representation of $B$ than in the logical matrix representation of $A$. As a consequence, these additional 1 s may be viewed as the outcome of the $D_{A B}$, a matrix addition. After that, we obtained the following one-to-one functor:

$$
\begin{aligned}
& \psi: \text { PS } \rightarrow \text { LM } \\
& A \mapsto \psi(A) \\
& B \mapsto \psi(B) \\
& \psi: \operatorname{Hom}_{\mathbf{P S}}(\mathrm{A}, \mathrm{B}) \mapsto \operatorname{Hom}_{\mathbf{L M}}(\psi(A), \psi(B)) \\
& f \mapsto \psi(f)=D_{A B}
\end{aligned}
$$

The one-to-one property of functor $\psi$ in Formula (2) comes after the discussion in the last section. A one-to-one mapping with a middle state characterizes a logical matrix representation. 


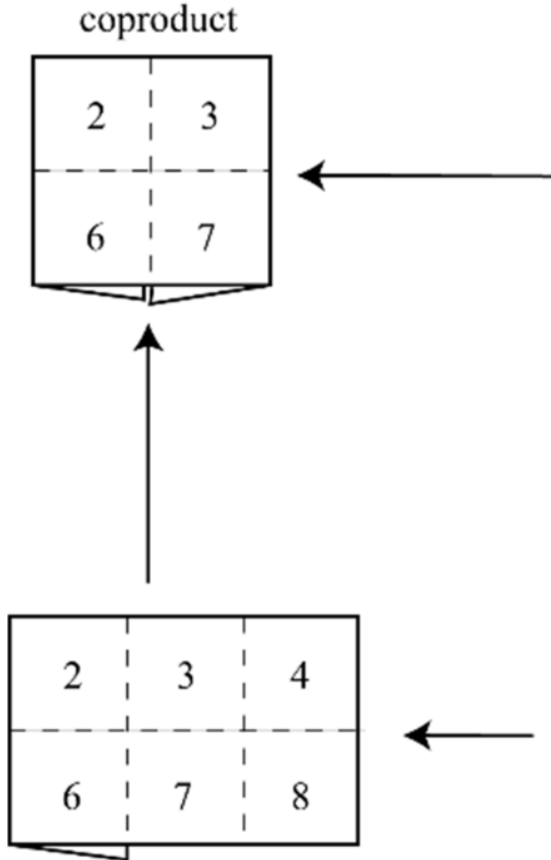

B
A
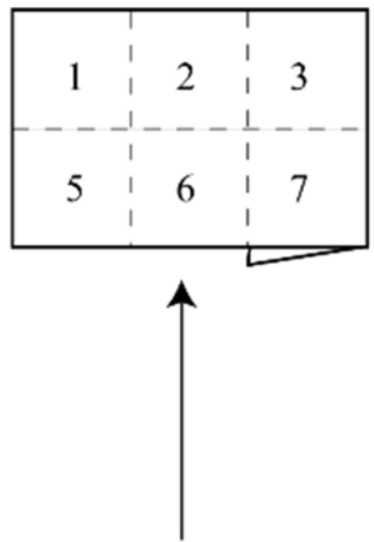

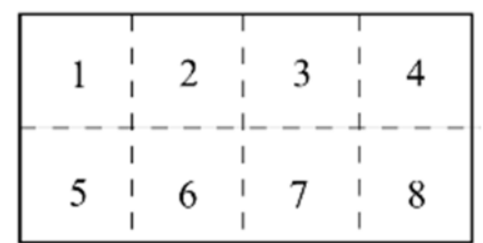

product

Figure 4. Product and coproduct of two middle states $A$ and $B$.

\subsection{Specifications}

Now, we can discuss the categorical conceptions in these two categories.

(1) Initial object and terminal object: Both categories have only initial objects but no terminal objects. The initial objects are the initial unfolded state of the map $M$ and the zero matrices, respectively. They have no terminal object because $M$ can have multiple final folded states.

(2) Opposite category: Turn over the arrows in these two categories, and we get the opposite categories. The opposite category of PS, denoted $\mathbf{P S}^{\mathbf{o p}}$, takes the middle states of the map during a simple folding as its objects, and takes the inverse operation, simple unfolds, as its morphisms. Note that even though we can define the opposite category of $\mathbf{L M}$ by using "subtraction-like" morphisms, the operation cannot be naturally derived from the operations over the semiring $\Omega$. This fact indicates the particularity of simple folds.

(3) Direct system: Each simple folding derives a direct system in PS. The general definition of a direct system is presented as: Let $(I, \leq)$ be a directed index set and let $A_{i}: i \in I$ be a family of indexed objects. If a homomorphism $f_{i j}: A_{i} \rightarrow A_{j}$ for all $i \leq j$ satisfies that $f_{i i}$ is the identity morphism and $f_{i k}=f_{j k} \cdot f_{i j}$ for any triple $i \leq$ $j \leq k$, then $\left(A_{i}, f_{i j}\right)$ comprises a direct system. Take the middle states during a certain simple folding as $A_{i}$, take the simple folds which fold an earlier middle state $A_{i}$ to a latter middle state $A_{j}$ as $f_{i j}$, and take the empty folding identity morphism as $f_{i i}$, we can obtain a direct system in PS. It also develops a direct system in LM via the functor $\psi$. In $\mathbf{L M}$, the logical matrix representations of the middle states 
during a certain simple folding are taken as $A_{i}$ while the difference between the logical matrix representation $A_{i}$ and $A_{j}$ is taken as $f_{i j}$. The zero matrix is taken as $f_{i i}$.

(4) Product and coproduct: In PS, any two objects that represent two middle states which can be folded to a common state has a coproduct. The coproduct represents their nearest common middle state they can be simple-folded to. On the opposite, their product represents their nearest common middle state they can be simpleunfolded to. Because every two middle states can finally be simple-unfolded to the initial state of the map, products must exist. The illustration in figure 4 shows an example, where the product of two middle states $A$ and $B$ is the initial state of the map and the coproduct of them is their nearest further folded state. Moreover, the products and coproducts are alternated in its opposite category $\mathbf{P S}^{\mathbf{o p}}$. Using the functor $\psi$, we can obtain the products and coproducts in LM.

\section{Conclusion and Future Work}

We addressed how to obtain the category of the middle states while simple-folding a map using the logical matrix representation as an intermediate in this work. We studied two categories and confirmed the most prevalent conceptions in the category theory. Moreover, we proposed a highly abstracted model for the simple-folding and pointed out its potential to be applied to general flat-foldings.

Our future work will focus on how to extend the categorical analysis to flat or even $3 \mathrm{D}$ foldings, as well as how to apply our categorical description to solve specific problems.

\section{Acknowledgments}

This work was supported by the National Natural Science Foundation of China (No.61902155) and Research Initiation Fund for Senior Talents of Jiangsu University (No.19JDG024).

\section{References}

[1] Webb P 2007 An introduction to the representations and cohomology of categories. Group representation theory $149-73$.

[2] Bredon G E 2012 Sheaf theory (Vol. 170). Springer Science \& Business Media.

[3] Heunen C, Vicary J 2019 Categories for Quantum Theory: an introduction. Oxford University Press.

[4] Arkin E M, Bender M A, Demaine E D, Demaine M L, Mitchell J S, Sethia S, Skiena S S 2004 When can you fold a map? Computational Geometry 29(1) 23-46.

[5] Uehara R 2016 Stamp foldings with a given mountain-valley assignment. In Origami 5: Proceedings of the 3rd International Meeting of Origami Science, Math, and Education (pp. 599-612). AK Peters/CRC Press.

[6] Bern M, Hayes B 1996 January The complexity of flat origami. In SODA (Vol. 96, pp. 175-83).

[7] Morgan T D 2012 Map folding (Master's dissertation, Massachusetts Institute of Technology).

[8] Hull T 2002, July The combinatorics of flat folds: a survey. In Origami3: Proceedings of the 3rd International Meeting of Origami Science, Math, and Education (pp. 29-38). 
[9] Jia Y, Mitani J and Uehara R 2021 Research on Map Folding with Boundary Order on Simple Fold. IEICE Transactions on Fundamentals of Electronics, Communications and Computer Sciences, 2020 DMP0017.

[10] Abdali S K, Saunders B D 1985 Transitive closure and related semiring properties via eliminants Theoretical Computer Science 40 257-74. 